\title{
A Multi-Channel Stimulator With High- Resolution Time-to-Current Conversion for Vagal-Cardiac Neuromodulation
}

\author{
Yu Wu, Member, IEEE, Dai Jiang Senior Member, IEEE, and Andreas Demosthenous, Fellow, IEEE
}

\begin{abstract}
This paper presents a low power integrated multichannel stimulator for a cardiac neuroprosthesis designed to restore the parasympathetic control after heart transplantation. The proposed stimulator is based on time-to-current conversion. It replaces the conventional current mode digital-to-analog converter (DAC) that uses tens of microamps for biasing, with a novel capacitor time-based DAC (CT-DAC) offering about 10-bit current amplitude resolution with a bias current of only $250 \mathrm{nA}$. A stimulator chip was designed in a $0.18 \mu \mathrm{m}$ CMOS high-voltage (HV) technology. It consists of 16 independent channels, each capable of delivering up to $550 \mu \mathrm{A}$ stimulus current with a $\mathrm{HV}$ output stage that can be operated up to $20 \mathrm{~V}$. The stimulator chip performance was evaluated using both $\mathrm{RC}$ equivalent load and a microelectrode array in saline solution. It is power efficient, provides high-resolution current amplitude stimulation, and has good charge balance. The design is suitable for multi-channel neural stimulation applications.
\end{abstract}

Index Terms-Digital-to-analog converter (DAC), heart transplant, high voltage neural stimulator, implantable devices, time-to-current converter, vagus nerve stimulation.

\section{INTRODUCTION}

$\mathrm{E}$ very year over 3000 heart transplantation surgeries are conducted worldwide. Surgery is the last resort for a patient who is at the end-stage of heart failure. Because of the complexity involved during the surgery, denervation is inevitable and causes the transplanted heart to function without any parasympathetic control. By innervating the sinoatrial and atrioventricular nodes, the parasympathetic control functions to slow the heart rate and relax the heart [1]. Parasympathetic control is facilitated by the vagus nerve for cardiac activity modulation; denervation means this connection is lost between the patient's vagus nerve and the donor heart which is only controlled by the circulation catecholamines. Therefore, even though heart transplantation can extend the life expectancy of patients, health-related problems remain due to cardiac denervation. Related chronotropic incompetence not only affects the exercise capacity and health-related quality of life but also leads to long-term complications.

To re-bridge this neural link, a cardiac neuroprosthesis is required as shown in the conceptual diagram in Fig. 1. Here, an

Manuscript received August 30, 2021; revised November 11, 2021.

This work was supported by the European Union's Horizon 2020 Research and Innovation Program under Grant 824071.

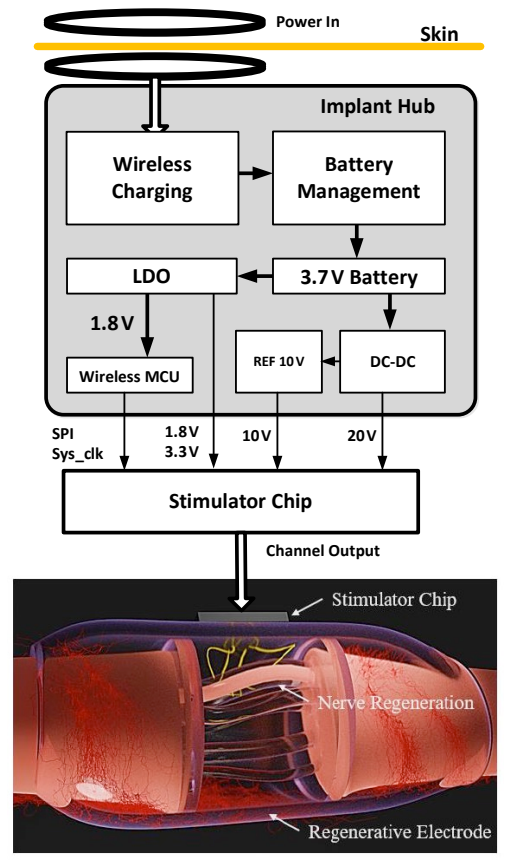

Fig. 1. Concept of cardiac neuroprosthesis with a regenerative active electrode array for vagal-cardiac reconnection.

active regenerative electrode with a multi-channel stimulator chip for artificial vagus control is proposed. The regenerative neural interface is designed to provide guided nerve regeneration [2], [3]. Once the nerve is regenerated, the neuroprosthesis could facilitate cardiac-vagal re-connection by providing multi-site stimulation. With both the re-connection of the vagus nerve and 'grafting' of the electrode stimulation capability, the parasympathetic control would be reinstated.

The entire system consists of an implant hub providing power and communication to the active site where the stimulator chip is located, as shown in Fig. 1. This paper focuses on the design of the neural stimulator chip. A simple time-to-current digitalto-analog (DAC)-based stimulator is proposed. The stimulator has a time-to-current conversion rate of $74.2 \mathrm{nA}$ per DAC clock cycle, controlled by a $15 \mathrm{MHz}$ timing clock achieving about 10bit DAC resolution. With four current scaling options, it can

The authors are with the department of Electronic and Electrical Engineering, University College London, Torrington Place, WC1E 7JE London, U.K. (e-mail: yu.wu.09@ucl.ac.uk; d.jiang@ucl.ac.uk; a.demosthenous@ucl.ac.uk). 


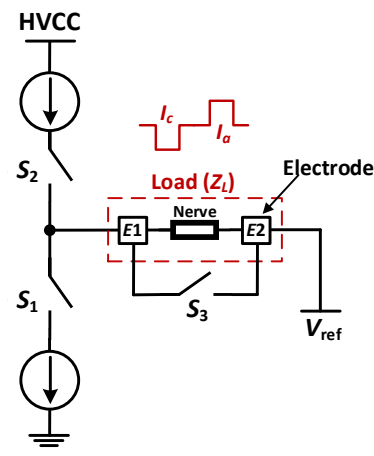

(a)

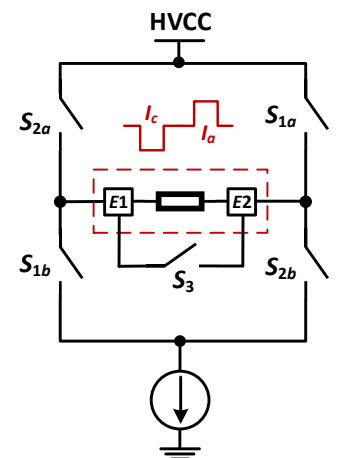

(b)
Fig. 2. Stimulator output stage topologies for generating biphasic current pulses $\left(I_{a}\right.$ and $\left.I_{c}\right)$. (a) Using a source-sink current driver pair. (b) Using a single current driver with an H-bridge.

output a maximum stimulus current $\left(I_{\text {stim }}\right)$ of $550 \mu \mathrm{A}$ from a $20 \mathrm{~V}$ high-voltage (HV) supply. The DAC uses only $250 \mathrm{nA}$ biasing current. The total power consumption during biphasic stimulation is dynamic but the static power is only $23.3 \mu \mathrm{W}$.

The proposed 16-channel time-to-current stimulator design provides high resolution stimulus currents with independent channel control. It is also power efficient and scalable for multichannel stimulation. The rest of the paper is organized as follows. Section II outlines the stimulator design considerations and Section III describes the circuit details of the various system blocks. Section IV presents measured results from the fabricated stimulator chip implemented in a $0.18 \mu \mathrm{m}$ CMOS $\mathrm{HV}$ technology, including testing with a cardiac microelectrode array in saline solution. Concluding remarks are drawn in Section V. The paper expands on the preliminary design reported in [4].

\section{Stimulator Design Considerations}

A conventional constant current neural stimulator fundamentally consists of a programmable current driver. It delivers current pulses between a pair of electrodes connected to the targeted nerve where neural responses are elicited by a stimulus current ranging from tens of microamperes $(\mu \mathrm{A})$ to tens of milliamperes (mA) [5]. Numerous integrated stimulator designs have been reported; they typically comprise of two main parts:

(i) A DAC to generate the desired current amplitude. This often uses a current mirror-based DAC (I-DAC) whose output current is multiplied/mirrored to the output stage for biphasic stimulation [6].

(ii) An output stage that connects to the electrodes for stimulation. There are two common approaches to generate biphasic current stimulation [7]; either a source-sink current driver pair [8] as shown in Fig. 2(a), or a single current driver with an H-bridge [9] as in Fig. 2(b).

An implantable stimulator must account for the following two design considerations [10]:

1) Low Power Consumption - Over the years, with an increasing demand for spatial resolution in neural interface microsystems, the number of electrodes has been increasing while the size of each electrode has been significantly

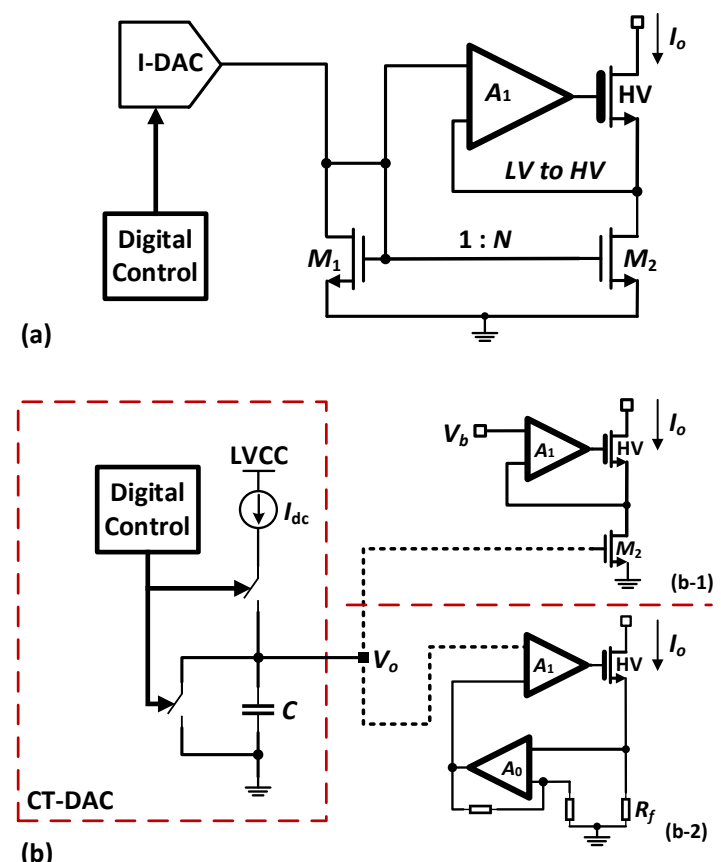

Fig. 3. (a) Conventional stimulator based on I-DAC. (b) Proposed time-tocurrent converter stimulator topology using CT-DAC; (b-1) Design option 1, (b-2) Design option 2.

decreasing. This leads to unavoidable high load impedances (in the region of tens of $\mathrm{k} \Omega$ ) that the stimulator must drive [11]. Hence, the output stage of the stimulator is often designed with HV transistors or low-voltage (LV) transistor stacks [12], [13] to provide enough voltage compliance. Given the strict power constraints imposed on implantable devices, state-of-the-art neural integrated stimulators are required to drive multiple channels and operate power-efficiently. For example, using dynamic HV supplies [14] or merging the I-DAC directly into a HV output stage at the expense of chip area [15], [16].

Although the HV output stage other requires unavoidable high-power consumption, power optimization can be achieved in the LV stage. A conventional high-resolution I-DAC design, for example, a 10-bit I-DAC with a $250 \mathrm{nA}$ LSB would consume up to $256 \mu \mathrm{A}$. In addition, the transistors in the layout must be scaled up from the minimum size transistor by a maximum of 1024. Allocating one DAC per stimulator channel in a multi-channel design is challenging. Thus, for multichannel stimulators either DAC sharing is adopted [6] or multiple low-resolution ( $\leq 8$-bit) DACs are often employed [14], [17]-[21] and calibration may be required to achieve good DAC performance [22].

2) Charge Balance - For safe stimulation a balanced biphasic current pulse is required; the total charge injected by the cathodic current phase must equal to the total charge removed by the anodic current phase. Any excess residual charge which builds up on the neural interface would potentially not only cause electrolysis at the electrodes but also damage the tissue [23].

Using the single current driver (which requires only one HV transistor) with an H-bridge [Fig. 2(b)] to generate biphasic current pulses provides good current matching characteristics and is simple to implement. However, for multi-channel 
Current Amplitude Scaling

\begin{tabular}{|ccc|}
\hline$i S_{2}$ & $i S_{1}$ & Factor \\
\hline 0 & 0 & $\times 1$ \\
0 & 1 & $\times 3$ \\
1 & 0 & $\times 5$ \\
1 & 1 & $\times 7$ \\
\hline
\end{tabular}

Biphasic Operation

\begin{tabular}{|cccc|}
\hline$S_{\mathrm{HV} 1}$ & $S_{\mathrm{HV} 2}$ & $i S_{4}$ & Phase \\
\hline$R$ & Open & Closed & Cathodic \\
$L$ & Closed & Open & Anodic \\
\hline
\end{tabular}
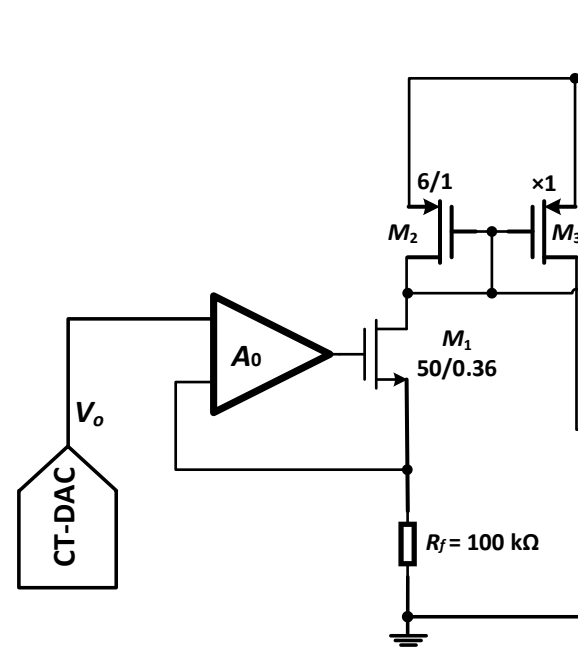

$\stackrel{\text { LVCC }}{T}$

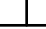

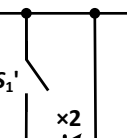
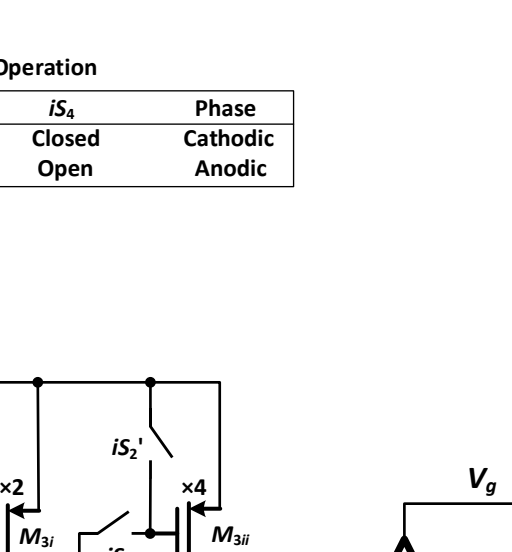

HVC

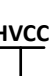

CC

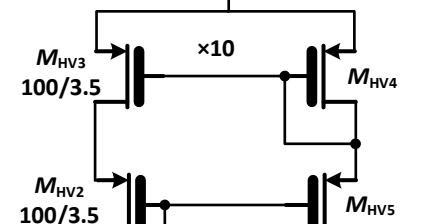

$100 / 3.5$
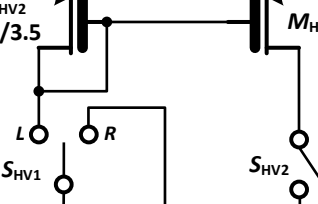

10

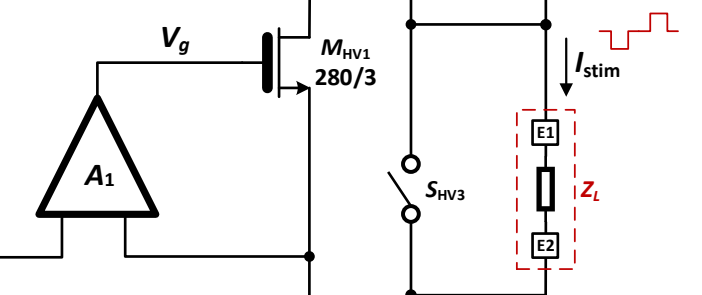

Fig. 4. The output stage of the neural stimulator. $M_{\mathrm{HV}}$ transistors and $S_{\mathrm{HV}}$ switches are high voltage. All other devices are low voltage.

stimulation in order to reduce electric crosstalk, time multiplexing [24] or power isolation [25] is required.

The source-sink topology [Fig. 2(a)] is more suitable for driving multiple channels, but it suffers from CMOS fabrication induced current mismatches which limit the resolution of the DAC. Electrode discharging is often required to remove any residual charge by either actively sending extra short-burst current pulses or temporarily passively shorting the electrodes together [facilitated by switch $S_{3}$ in Fig. 2(a) and (b)] after each biphasic pulse for better charge balancing [26].

\section{Stimulator Design}

\section{A. Voltage-to-Current Converter}

The proposed design employs a new DAC principle that is power efficient, simple to implement and scalable for multichannel stimulators. In the conventional design shown in Fig. 3(a), from a I-DAC a desired current amplitude is generated and then scaled up by the current mirror (LV transistors $M_{1}$ and $M_{2}$ ) to generate $I_{o}$. For HV operation, a cascode HV transistor under the bias control of opamp $A_{1}$ is added. This active feedback clamps the drain voltage of $M_{2}$, and not only provides reliable current mirroring and high output impedance, but also couples the LV MOS to the HV MOS to drive a large impedance load.

An alternative is shown in Fig. 3(b). By controlling the charging period of a capacitor, the desired voltage, previously supplied by a conventional I-DAC with multiple current branches, can instead be generated by simply charging the capacitor with a small constant dc current $\left(I_{\mathrm{dc}}\right)$, hence implementing a capacitor-charge time-based DAC (CT-DAC). In the CT-DAC, the relationship between charging time and voltage developed across the capacitor is essentially linear. The time-to-current control can be quite simple if the voltage-tocurrent conversion also has a linear relationship.

There are several options to couple the output voltage $\left(V_{o}\right)$ of the CT-DAC to the stimulator output stage:

(i) As shown in Fig. 3(b-1) by directly using the output voltage from the CT-DAC to bias, for example, the gate of transistor $M_{2}$ offers a more power efficient design than Fig. 3(a). If $M_{2}$ is biased in the triode region, the circuit is effectively a voltage-to-current converter with a linear transfer function of $I=V_{b} / R_{M_{2}}$, where $V_{b}$ is a fixed bias voltage and $R_{M_{2}}$ is the equivalent resistance of $M_{2}$ which varies according to the output voltage $V_{o}$ of the CT-DAC. The non-linearity of the MOS resistor $M_{2}$ is an inaccuracy requiring extra compensation [27].

(ii) A method to implement a voltage-to-current converter with better linearity is shown in Fig. 3(b-2) [4]. It uses a fixedvalue resistor $R_{f}$ instead of MOS resistor and applies the CTDAC voltage to the active feedback opamp $A_{1}$. This option has a linear output current transfer function:

$$
I_{o}=V_{o} \frac{g_{m_{\mathrm{HV}}} A_{o l}}{1+g_{m_{\mathrm{HV}}} A_{o l} G R_{f}} \approx \frac{V_{o}}{G R_{f}}
$$

where $g_{m_{\mathrm{HV}}}$ is the transconductance of the $\mathrm{HV}$ transistor, $A_{o l}$ is the open loop gain of $A_{1}$ and $G$ is the gain of the noninverting amplifier using opamp $A_{0}$. With the non-inverting amplification, the clamped source voltage of the HV transistor can be $G$ times smaller than $V_{o}$, offering higher voltage compliance but at the expense of power consumption due to the added opamps to drive the HV transistor.

(iii) An improved implementation is shown in Fig. 4. By placing the voltage-to-current converter on the LV side, the source of transistor $M_{1}$ can be clamped at higher voltage levels 


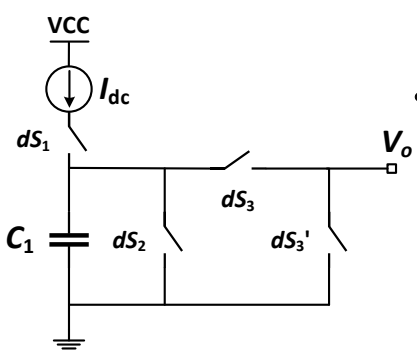

(a)

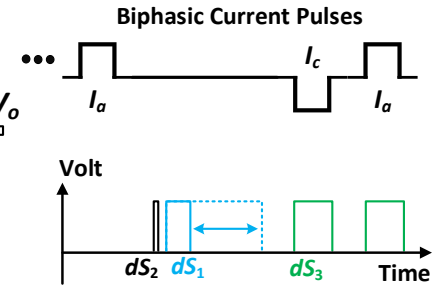

(b)
Fig. 5. (a) One capacitor implementation of CT-DAC. (b) Switch timing diagram.

[compared to Fig. 3(b-2)], providing a wider dynamic range for the CT-DAC. A simple feedback loop has a wider bandwidth and moderate power consumption. $R_{f}$ can be large (e.g., $100 \mathrm{k} \Omega$ ) for low-power operation. The drain current of $M_{1}$ can be scaled by different factors with the intermediate stage $\left(M_{2}, M_{3}, M_{3 i}, M_{3 i i}\right)$ according to the 'current amplitude scaling' table in Fig. 4.

To generate the cathodic phase of a biphasic pulse, the HV switch $S_{\mathrm{HV} 1}$ is connected to position $R$, and transistors $M_{5}$ and $M_{5 P}$ (with $i S_{4}$ closed) provide the cathodic current $\left(S_{\mathrm{HV} 2}\right.$ is open). The current flows from the common return electrode $E 2$ to electrode $E 1$. For the anodic phase, $S_{\mathrm{HV} 2}$ is closed, $S_{\mathrm{HV} 1}$ is changed to position $L$, and the HV PMOS current mirror $\left(M_{\mathrm{HV} 2}-M_{\mathrm{HV} 5}\right)$ provides the anodic current from $E 1$ to $E 2$. In the anodic phase, the gate of $M_{5 P}$ is grounded (by switching on $i S_{4}{ }^{\prime}$ ), and the drain current of $M_{\mathrm{HV} 1}$ is equal to that of $M_{4}$. It is scaled up by a factor of 10 by the $M_{\mathrm{HV} 2}-M_{\mathrm{HV} 5}$ current mirror. This is used to save power in the $M_{\mathrm{HV} 1}$ branch during the anodic phase. To discharge the electrodes in the discharge phase, $S_{\mathrm{HV} 3}$, $i S_{3}$ and $i S_{4}{ }^{\prime}$ are closed.

As an example, for a maximum cathodic current of $500 \mu \mathrm{A}$, with a scaling factor of $\times 70\left(M_{2}\right.$ to $M_{3} / M_{3 i} / M_{3 i i}$ times $M_{4}$ to $\left.M_{5} / M_{5 P}\right), M_{1}$ is required to conduct a drain current of $7.15 \mu \mathrm{A}$. It is sized to account for the maximum voltage provided by opamp $A_{0}(<1.8 \mathrm{~V})$ to provide $715 \mathrm{mV}$ across $R_{f}=100 \mathrm{k} \Omega$. The same consideration applies to the sizing of $M_{\mathrm{HV} 1}$; it is required to support $500 \mu \mathrm{A}$ under a limited gate-to-source voltage. Voltage $V_{g}$ provided by $A_{1}$ is limited by the available 3.3 V supply. The source voltage of $M_{\mathrm{HV} 1}$ is determined by the sizing of $M_{5}$ and $M_{5 P}$. Other considerations in relation to the sizing of the transistor include the available voltage, voltage compliance and layout area. The HV transistor dimensions are labeled in Fig. 4. Note that the maximum stimulator output current is also determined by the maximum output voltage $\left(V_{o}\right)$ provided by the CT-DAC.

\section{B. Time-to-Voltage Converter}

The basic CT-DAC circuit implementation is shown in Fig. 5(a) [4] with the corresponding digital control timing diagram in Fig. 5(b). There are two issues with this implementation:

(i) For asymmetrical biphasic pulses (beneficial for stimulation efficacy [19]), the charge on the capacitor in the CT-DAC must be recharged during the interphase delay. For short interphase delays this may be difficult.

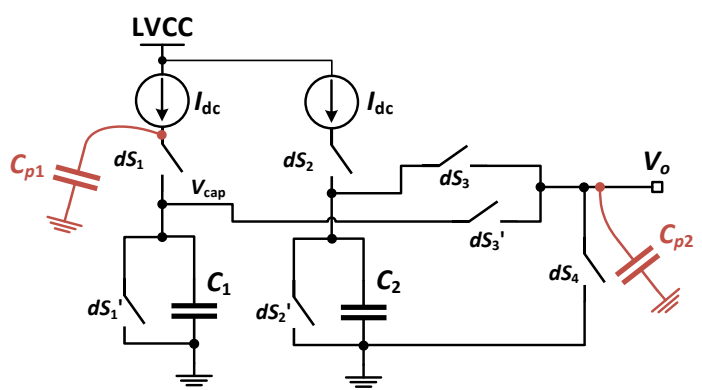

(a)

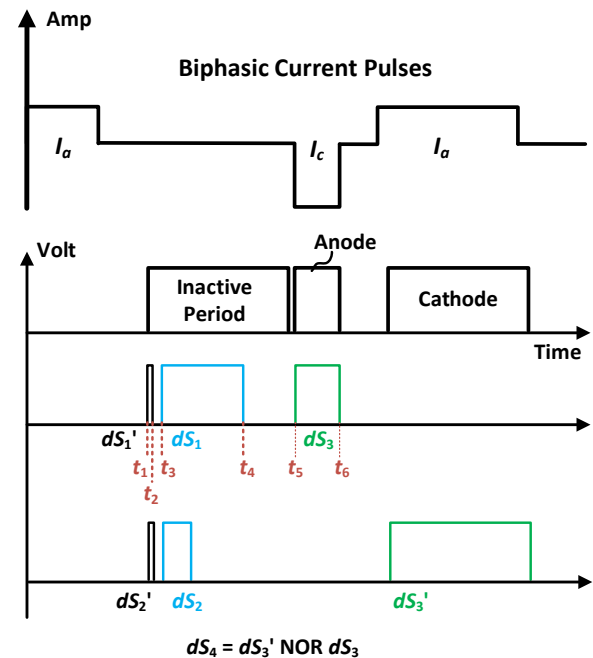

(b)

Fig. 6. (a) Implementation of improved CT-DAC with two capacitors. (b) Switch timing diagram.

(ii) The voltage-to-current converter in Fig. 3(b-2) used in [4] may need its input reset to ground (depends on its bandwidth) by closing $d S_{3}{ }^{\prime}$ during the pulse interphase delay. Hence, charge sharing between the CT-DAC capacitor and the input parasitic capacitance of opamp $A_{1}$ happens twice, resulting in different $V_{o}$ for a supposedly equal biphasic current output, lowering the bit resolution.

An improved CT-DAC design is shown in Fig. 6(a). It uses two capacitors that can be charged to different voltage levels for asymmetrical biphasic current outputs with the same dc current $I_{\mathrm{dc}}$. Fig. 6(b) shows the timing control diagram. After $d S_{1}{ }^{\prime}$ and $d S_{2}{ }^{\prime}$ reset capacitors $C_{1}$ and $C_{2}$, they can be charged to different voltages according to the ON times of $d S_{1}\left(T_{d S_{1}}\right)$ and $d S_{2}\left(T_{d S_{2}}\right)$ during the inactive period. Then $d S_{3}$ and $d S_{3}{ }^{\prime}$ select which voltage is applied to opamp $A_{0}$. The overall digital system operates under a $1 \mathrm{MHz}$ master clock, and the charging period is controlled by another CT-DAC clock. In this design, the capacitor was $25 \mathrm{pF}$ and the charging current $I_{\mathrm{dc}}$ was $250 \mathrm{nA}$. This is the total bias current required for the CT-DAC. The voltage developed across each capacitor is given by:

$$
V_{o}=I_{\mathrm{dc}}\left(T_{d S_{i}} / C_{i}\right)
$$

For example, consider a $15 \mathrm{MHz}$ CT-DAC clock with a 10bit current amplitude counter. In each clock cycle the capacitor 


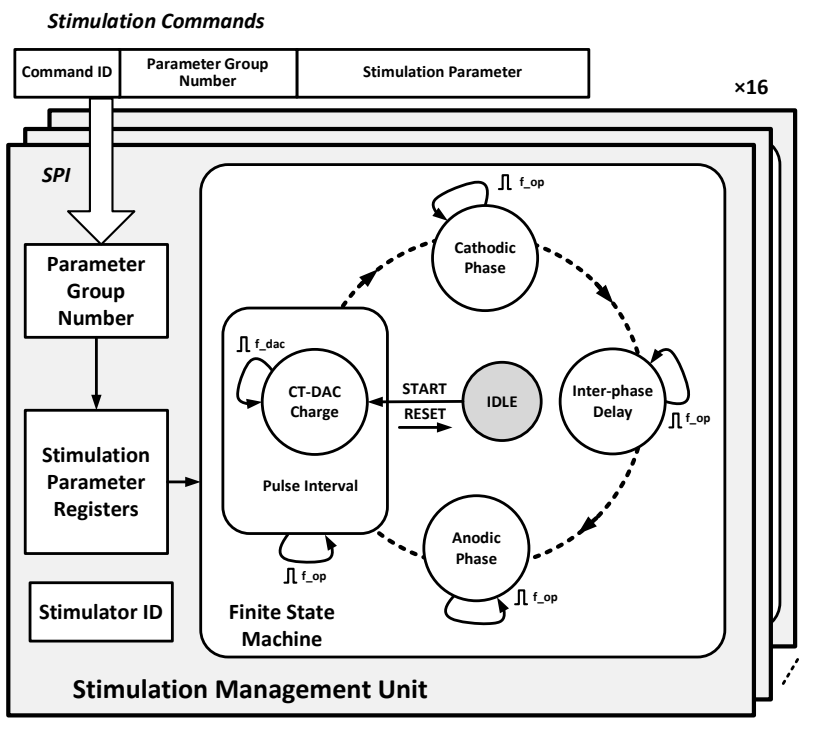

Fig. 7. The digital control for stimulator multi-channel management.

charges up by $667 \mu \mathrm{V}$, leading to a maximum output voltage $V_{o}$ of $682 \mathrm{mV}$. For $R_{f}=100 \mathrm{k} \Omega$, the drain current of $M_{1}$ is $6.82 \mu \mathrm{A}$, and with a scaling factor of $\times 70$ yields a maximum stimulator output current of $478 \mu \mathrm{A}$. This translates to an LSB output current of $66.7 \mathrm{nA}$ per CT-DAC clock cycle (the other 3 scaling factors shown in Fig. 4 provide different LSB output current values). In addition, other CT-DAC clock frequencies can be used leading to different LSB values, hence simple adjustment of the current resolution.

Charge injection and charge sharing will affect the voltage developed on the capacitor $\left(V_{\text {cap }}\right)$ in the CT-DAC. This can be examined using the timing diagram in Fig. 6(b):

(i) Before $t_{1}, d S_{1}{ }^{\prime}$ and $d S_{1}$ are open, and the parasitic capacitor $C_{p 1}$ shown in Fig. 6(a) is charged to LVCC.

(ii) From $t_{1}$ to $t_{2}, d S_{1}{ }^{\prime}$ resets the voltage on $C_{1}$ to ground and opens at $t_{2}$. As $d S_{1}{ }^{\prime}$ opens, the charge injection $\Delta Q$ from $d S_{1}{ }^{\prime}$ is transferred to $C_{1}$. It is approximately:

$$
\begin{gathered}
\Delta Q \approx \frac{1}{2}\left[W_{n} L_{n} C_{\text {ox }}\left(V_{\text {control }}-V_{\text {in }}-V_{t h n}\right)\right. \\
\left.-W_{p} L_{p} C_{\text {ox }}\left(V_{\text {in }}-\left|V_{t h p}\right|\right)\right]
\end{gathered}
$$

where $V_{\text {control }}$ is the control signal applied to the gate of the switch, $V_{\text {in }}$ is the input signal to the switch, and the other symbols are the conventional notations for a standard CMOS transmission gate switch. As shown, $\Delta Q$ is $V_{\text {in }}$ dependent; in simulation, when $V_{\text {in }}$ of $d S_{1}{ }^{\prime}$ is $0 \mathrm{~V}$ at $t_{2}, \Delta Q_{d s_{1^{\prime}}}=3.5 \mathrm{fC}$. This charge injection will be the same for all the CT-DAC charging cycles.

(iii) As $d S_{1}$ closes at $t_{3}$, charge sharing happens between $C_{1}$ and the parasitic capacitor $C_{p 1}$. Hence, prior to any current charging, there will be an initial voltage on $C_{1}$ due to $\Delta Q_{d s_{1}}$, and the stored charge on $C_{p 1}$. The initial voltage is constant for all the charging cycles, and in simulation, this voltage was around $2.5 \mathrm{mV}\left(C_{1}=25 \mathrm{pF}\right)$.

(iv) As $d S_{1}$ opens after the charging of the CT-DAC is completed at $t_{4}$, its charge injection $\Delta Q_{d S_{1}}$ is transferred to $C_{1}$. From (3) it can be seen that a net-zero $\Delta Q$ occurs at $V_{q 0}=$ $V_{\text {control }} / 2$ ideally, and as $V_{\text {in }}$ moves away from $V_{q 0}$ (which

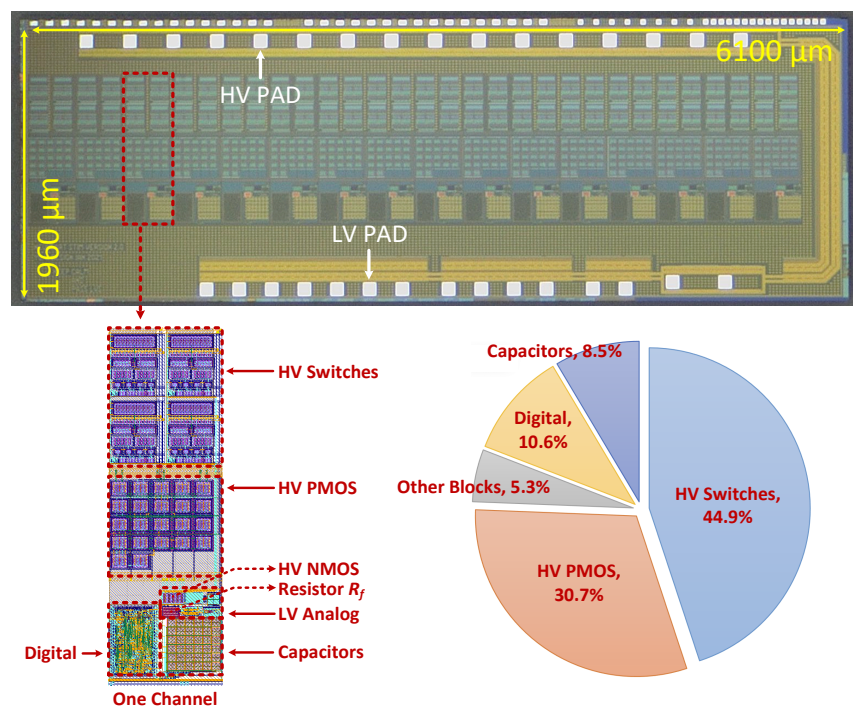

Fig. 8. Stimulator chip microphotograph and details of a single channel. The layout area breakdown is also shown. 'Other blocks' include the low-voltage analog circuits, feedback resistor $R_{f}$ and HV NMOS $M_{\mathrm{HV} 1}$.

from simulation was around $0.5 \mathrm{~V}) \Delta Q_{d s_{1}}$ increases. Hence, in any CT-DAC operation, one equivalent LSB charge on $C_{1}$ must be larger than $3.5 \mathrm{fC}$, which is the worst-case for $\Delta Q_{d s_{1}}$ when $V_{\text {in }}=0 \mathrm{~V}$. This is the only critical charge injection that must be accounted for in the design. Using a $15 \mathrm{MHz}$ CT-DAC clock, a charging current of $250 \mathrm{nA}$ for 10-bit resolution causes a LSB charge of $16.7 \mathrm{fC}$ ( 4.76 times $3.5 \mathrm{fC}$ ). The value of $C_{1}$ is dictated by the maximum CT-DAC output voltage for 10-bit resolution.

(v) When $d S_{3}$ closes at $t_{5}$, charge sharing happens between capacitors $C_{1}$ and parasitic capacitor $C_{p 2}$. As $d S_{4}$ always resets $C_{p 2}$ to ground, and the final CT-DAC output is $V_{o}=$ $C_{1} V_{\text {cap }} /\left(C_{1}+C_{p 2}\right)$. This does not affect the CT-DAC resolution because $C_{p_{2}}$ is the same for each stimulator channel. Mismatches, for example, between $C_{1}$ and $C_{2}$ can be minimized by careful layout design.

(vi) When $d S_{3}$ opens at $t_{6}$, its charge injection is no longer relevant. Subsequently a new cycle can begin.

The charge on capacitors $C_{1}$ and $C_{2}$ must ideally be held constant after $d S_{1}$ or $d S_{2}$ have been turned off to the end of its corresponding pulse duration (when $d S_{3}$ or $d S_{3}{ }^{\prime}$ turn off); see Fig. 6(b). Charge leakage should be considered. A $15 \mathrm{MHz}$ CTDAC of 10-bit resolution, results in a $68.2 \mu$ s inactive period. For a pulse width of $100 \mu \mathrm{s}$, when holding one LSB of charge the worst case is losing half the LSB on $C_{2}$ (at the end of the anodic phase) over $268 \mu \mathrm{s}$. This results in a maximum allowable leakage current of $Q_{\mathrm{LSB}} / t=0.5(16.7 \mathrm{fC} / 268 \mu \mathrm{s}) \approx$ $31.2 \mathrm{pA}$ for a $250 \mathrm{nA} \mathrm{CT}$-DAC charging current.

\section{Stimulator Digital Control}

For the targeted application requiring multi-channel stimulation, 16 stimulators were implemented on chip. Each stimulator is identified with a dedicated hardwired stimulator ID. The stimulators can operate individually, or several stimulators can be grouped together using the stimulator ID for synchronized stimulation. 


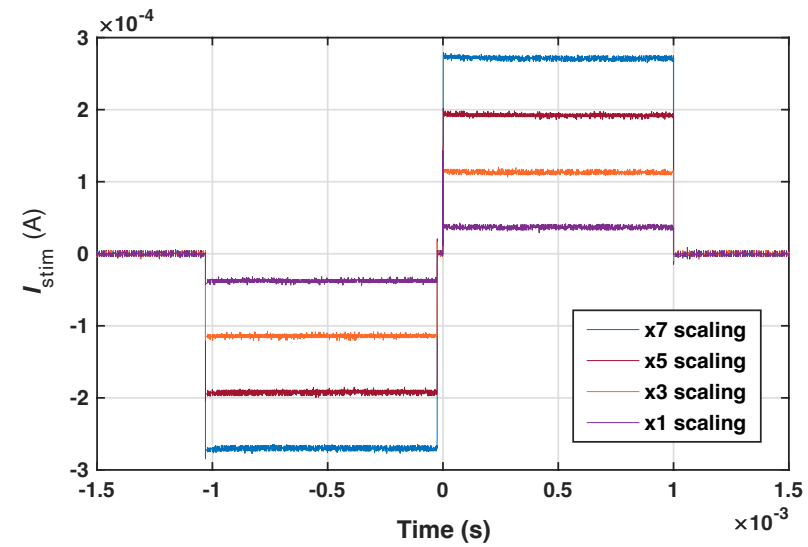

Fig. 9. Biphasic stimulator output current $\left(I_{\text {stim }}\right)$ with a $2 \mathrm{k} \Omega$ resistor load for different scaling factors.

Stimulation parameters are sent to all the stimulators via a single serial peripheral interface (SPI). Each SPI command frame is headed with a parameter group number and a command ID. Stimulators are firstly assigned to a group, and those with the matching group number operate with the set parameters, e.g., stimulator with ID-1, ID-5 and ID-13 can be grouped into stimulator group 1 and operate in parallel (the stimulation parameters of group 1 are sent by the SPI).

The stimulation management logic in each stimulator stores the parameters to specific registers according to the command ID to set the biphasic current amplitudes, pulse width, interphase delay, pulse interval and amplitude scaling factors. Once all the parameters are received, the finite-state machine (FSM) leaves the idle state to start periodic operations as illustrated in Fig. 7. The states in the FSM are controlled by cascaded counters.

\section{MEAsured Results}

The stimulator chip was implemented in XFAB $0.18 \mu \mathrm{m} \mathrm{HV}$ CMOS technology. The overall chip size is $1960 \mu \mathrm{m} \times 6100 \mu \mathrm{m}$, and each stimulator channel occupies an area of $350 \mu \mathrm{m} \times 1100 \mu \mathrm{m}$. The design uses the available $45 \mathrm{~V}$ HV MOS. The $3 \mathrm{HV}$ switches $(350 \mu \mathrm{m} \times 410 \mu \mathrm{m}$ in total $)$ and the 4 HV PMOS ( $350 \mu \mathrm{m} \times 280 \mu \mathrm{m}$ in total) occupy the largest area. The two capacitors in the CT-DAC occupy an area of $165 \mu \mathrm{m} \times 165 \mu \mathrm{m}$ in total. The other blocks include the lowvoltage analog circuits, feedback resistor $R_{f}$ and NMOS HV $M_{\mathrm{HV} 1}$. The chip microphotograph is shown in Fig. 8 with the percentage area breakdown shown in the pie chart.

\section{A. Chip Performance}

For chip performance tests, the stimulator was first connected to a resistor load of $2 \mathrm{k} \Omega$ and the voltage across it provided the stimulator output current. The CT-DAC clock was set to 15 $\mathrm{MHz}$. The selected stimulator channel was set to output half the maximum output current (amplitude counter set to 512) with a symmetrical pulse width of $1000 \mu$ s each phase, an interphase delay of $25 \mu \mathrm{s}$ and a pulse frequency of $100 \mathrm{~Hz}$.

The biphasic output current for different scaling factors is shown in Fig. 9. The measured output current with $\times 1$ scaling was $38 \mu \mathrm{A}$, resulting in a time-to-current conversion rate of
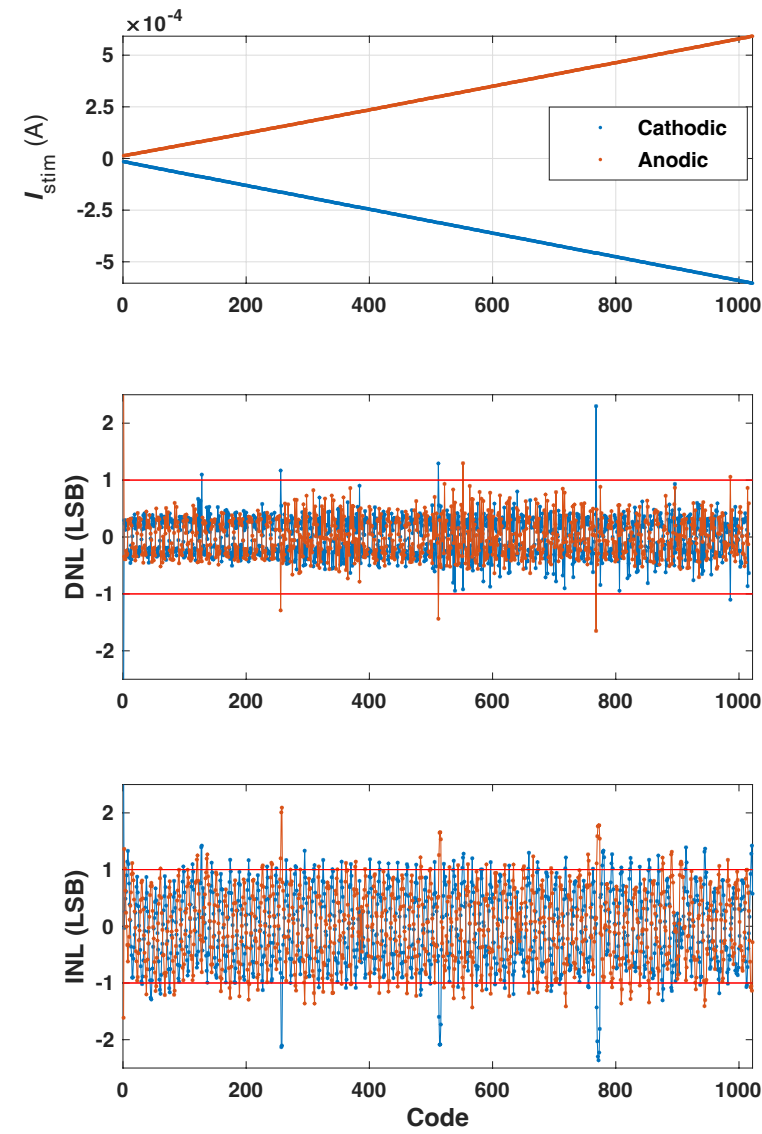

Fig. 10. The measured stimulator cathodic and anodic currents and their DNL and INL plots over a 10-bit digital sweep.

$74.2 \mathrm{nA}$ per clock, slightly higher than the design value (based on the $15 \mathrm{MHz}$ DAC counter clock) of $66.7 \mathrm{nA}$ per clock due to process variations. Other scaling factors were measured to be $\times 3.1, \times 5.2$ and $\times 7.3$, providing a maximum output current of $554.3 \mu \mathrm{A}$ at full range with the largest scaling factor.

The other important parameter in this design is the bit resolution provided by the CT-DAC implementation. It was measured over a 10-bit digital sweep. The DNL and INL of the CT-DAC was extrapolated from the measured voltage across the $2 \mathrm{k} \Omega$ resistor load. The measured cathodic and anodic currents together with the DNL and INL plots are shown in Fig. 10.

It is important to investigate the mismatch between the cathodic and anodic currents due to process variations. The measurements in Fig. 9 were repeated for each channel and the current mismatches are plotted in Fig. 11. On average the cathodic and anodic current mismatch is $3.86 \%$. This will lead to charge imbalance examined in the next section.

\section{B. Charge Balancing and Experiments With Electrodes in Saline}

For charge balancing evaluation, a $\mathrm{RC}$ electrode model ( $R_{x}=100 \mathrm{k} \Omega$ and $C_{x}=100 \mathrm{nF}$ in parallel and $R_{i}=2 \mathrm{k} \Omega$ in series) was connected in series with a sensing resistor $R_{s}=100 \Omega$. Due to imbalanced biphasic pulses, residual charge $\Delta Q_{r}$ remains on capacitor $C_{x}$. Without any intervention, 


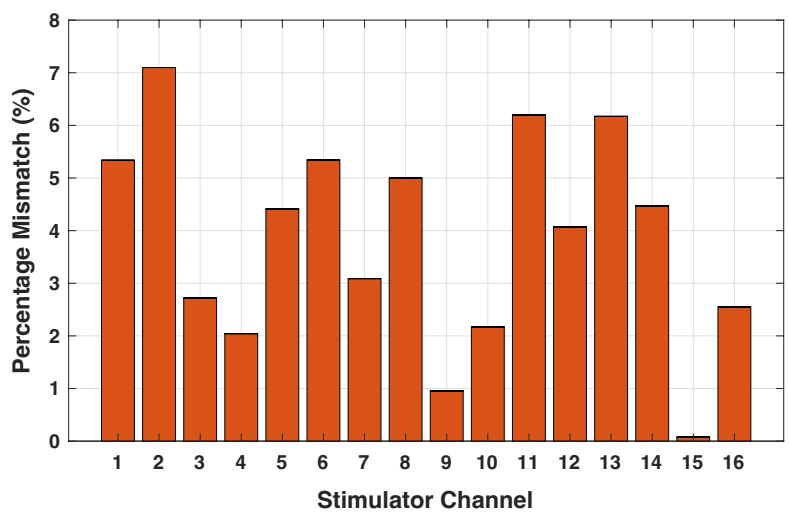

Fig. 11. Mismatch of the cathodic and anodic currents in each of the 16 independent channels in one stimulator chip. The amplitude of the cathodic and anodic currents was set to $200 \mu \mathrm{A}$.

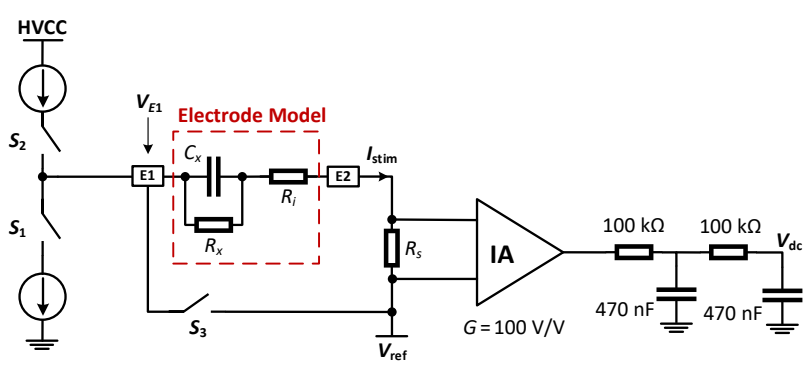

(a)

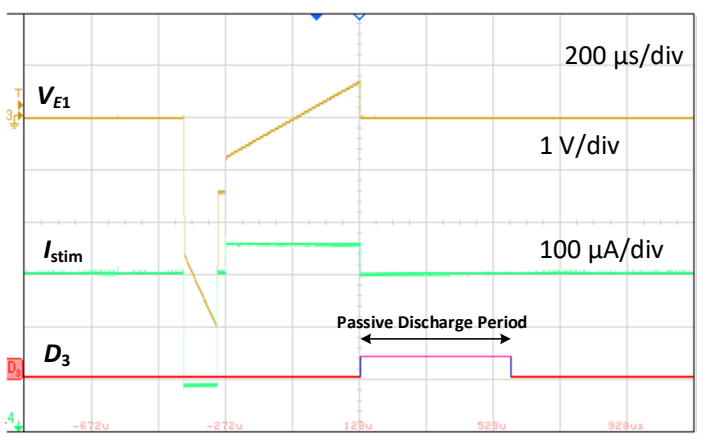

(b)

Fig. 12. (a) Setup for the residual dc measurements using the RC electrode model with passive discharge. (b) Voltage and current oscillograms of the asymmetrical biphasic stimulus pulses with the discharge switch timing. $V_{E 1}$ measured with a gain of 6 . The stimulation pulse repetition rate was $100 \mathrm{~Hz}$. $D_{3}$ is the digital pulse defining the passive discharge period when $S_{3}$ closes.

$\Delta Q_{r}$ naturally discharges through $R_{x}$ with a time constant $R_{x} C_{x}$ at the pulse frequency.

Passive discharge is a simple but effective method to provide a low impedance discharge path for quicker $\Delta Q_{r}$ removal. By closing switch $S_{3}$ in Fig. 12(a) (see $S_{\mathrm{HV} 3}$ in Fig. 4), the combination of $R_{\mathrm{on}}, R_{S}$ and $R_{i}$ where $R_{\mathrm{on}} \approx 750 \Omega$ is the on resistance of $S_{3}$, provides a shorter discharging time constant. The effectiveness of passive discharge can be application and electrode impedance dependent. In the targeted application (chronic vagus nerve stimulation), the recommended stimulation threshold is below $20 \mathrm{nC}$ for a pulse width of $100 \mu \mathrm{s}$ and a frequency of $1 \mathrm{~Hz}$ [28]. The recommended safety limit for the equivalent residual dc current should be less than $100 \mathrm{nA}$ [29]. Considering the above, the biphasic current pulse

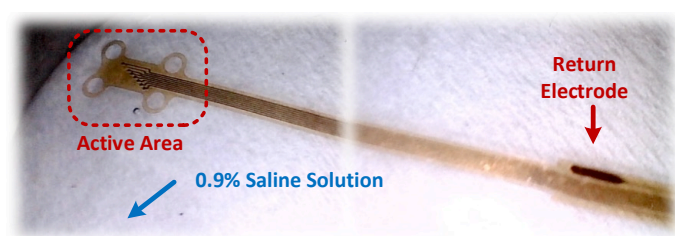

(a)

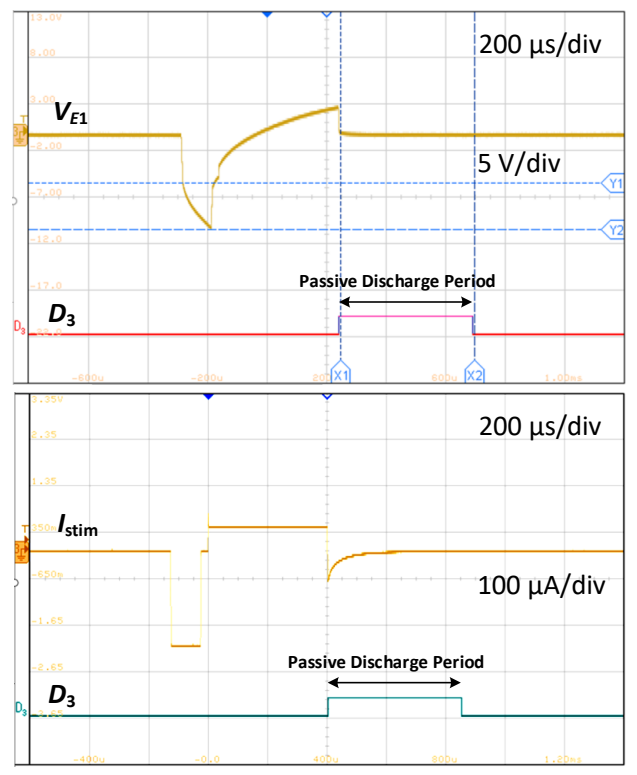

(b)

Fig. 13. (a) Microelectrode array immersed in saline solution. (b) Voltage and current oscillograms of the asymmetrical biphasic stimulus pulses with the discharge switch timing. $V_{E 1}$ measured with a gain of 6 . Identical anodic and cathodic currents were used to those in Fig. 12.

parameters were set as follows: cathodic current $200 \mu \mathrm{A}$, pulse width $100 \mu \mathrm{s}$, interphase delay $25 \mu \mathrm{s}$, anodic current $50 \mu \mathrm{A}$, pulse width $400 \mu \mathrm{s}$. The pulse frequency was set to $100 \mathrm{~Hz}$ instead of $1 \mathrm{~Hz}$ to better illustrate the charge balancing condition.

The stimulation channel used in the charge balancing test was number 8 in Fig. 11. From Fig. 12(a) the residual current error, $i_{\mathrm{dc}}=V_{\mathrm{dc}} / R_{S}$, can register the current mismatches from the stimulator. Prior to the insertion of a passive discharge period, the measured $\Delta Q_{r}\left(i_{\mathrm{dc}}=95.1 \mathrm{nA}, t=10 \mathrm{~ms}\right)$ was $951 \mathrm{pC}$. By inserting a passive discharge period of $450 \mu \mathrm{s}$ after each completed biphasic cycle as shown in Fig. 12(b), 79.3\% of the $\Delta Q_{r}$ should be removed (the passive discharge path had a RC time constant of $285 \mu \mathrm{s}$ ). The measured reduction with passive discharge was $74 \%$ corresponding to a residual $\Delta Q_{r}$ of $248 \mathrm{pC}$ or residual $i_{\mathrm{dc}}$ of $24.8 \mathrm{nA}$. Note that the remaining $\Delta Q_{r}$ will continue to discharge through $R_{x}$ until the next biphasic cycle; this would provide an additional residual error reduction.

The stimulator chip was also tested with a gold polyimide thin film electrode array in saline solution. The electrode array for vagus nerve stimulation is of the type shown in Fig. 13(a). It has eight $80 \mu \mathrm{m}$ diameter circular gold contacts on the active area and a long track extending to the back side where the larger return electrode is located. The entire electrode array was immersed in $0.9 \%$ saline solution; one active contact and the return electrode were connected to the ' $E 1$ ' and ' $E 2$ ' terminals shown in Fig. 12(a). The same asymmetric biphasic current in the RC electrode model experiment was used. The resulting 
Table I. Comparison with other stimulator designs.

\begin{tabular}{|c|c|c|c|c|c|c|c|}
\hline Reference & This work & [12] & [13] & {$[16]$} & [22] & [25] & [26] \\
\hline Technology & HV $0.18 \mu \mathrm{m}$ & $65 \mathrm{~nm} \mathrm{LP}$ & $\mathrm{LV} 0.18 \mu \mathrm{m}$ & HV $0.35 \mu \mathrm{m}$ & LV $0.18 \mu \mathrm{m}$ & $\mathrm{HV} 0.6 \mu \mathrm{m}$ & $\mathrm{LV} 0.18 \mu \mathrm{m}$ \\
\hline Stimulation channels & 16 & 4 & 16 & 1 & 8 & 2 & 1 \\
\hline Maximum current & $0.55 \mathrm{~mA}$ & $2 \mathrm{~mA}$ & $3 \mathrm{~mA}$ & $5.12 \mathrm{~mA}$ & $0.25 \mathrm{~mA}$ & $1 \mathrm{~mA}$ & $1 \mathrm{~mA}$ \\
\hline Output stage & Source-sink & H-bridge & Source-sink & Source-sink & Source-sink & $\begin{array}{l}\text { H-bridge } \\
\text { (chopped pulses) }\end{array}$ & H-bridge \\
\hline DAC design & $\begin{array}{l}\text { Independent } \\
\text { CT-DAC }\end{array}$ & $\begin{array}{l}\text { Independent } \\
\text { I-DAC }\end{array}$ & I-DAC sharing & $\begin{array}{l}\text { Independent } \\
\text { I-DAC }\end{array}$ & $\begin{array}{l}\text { Independent } \\
\text { I-DAC }\end{array}$ & I-DAC sharing & I-DAC \\
\hline DAC resolution & $\sim 10$-bit & 8-bit & 4-bit & 9-bit & 8-bit & 8-bit & 5-bit \\
\hline $\begin{array}{l}\text { Charge balance } \\
\text { strategy }\end{array}$ & Passive discharge & $\mathrm{N} / \mathrm{A}$ & Passive discharge & Active discharge & $\begin{array}{l}\text { Passive discharge } \\
\& \text { calibration }\end{array}$ & Passive discharge & $\begin{array}{l}\text { Passive discharge \& } \\
\text { Anodic modulation }\end{array}$ \\
\hline $\begin{array}{l}\text { Residual current }\left(i_{\mathrm{dc}}\right) \\
\text { or voltage }\left(V_{E 1)}\right.\end{array}$ & $3.9 \mathrm{nA}^{* 1}$ & $\mathrm{~N} / \mathrm{A}$ & $3.42 \mathrm{nA}^{* 2}$ & $\pm 20 \mathrm{mV}$ & $2-5 \mathrm{nA}^{* 3}$ & $5.5 \mathrm{nA}^{* 4}$ & $\pm 20 \mathrm{mV}^{* 5}$ \\
\hline Static power** & $23.3 \mu \mathrm{W}$ & $104 \mu \mathrm{W}$ & N/A & $31.8 \mu \mathrm{W}$ & N/A & N/A & $<0.59 \mathrm{~mW}$ \\
\hline Voltage compliance & $18.2 \mathrm{~V}$ & $\pm 11 \mathrm{~V}$ & $\pm 6 \mathrm{~V}$ & $22 \mathrm{~V}$ & $3.3 \mathrm{~V}$ & $12 \mathrm{~V}$ & $12.3 \mathrm{~V}$ \\
\hline Stimulator channel area & $0.385 \mathrm{~mm}^{2}$ & $0.36 \mathrm{~mm}^{2}$ & $0.08 \mathrm{~mm}^{2}$ & $1.88 \mathrm{~mm}^{2}$ & $0.064 \mathrm{~mm}^{2}$ & $<10 \mathrm{~mm}^{2}$ & $0.11 \mathrm{~mm}^{2}$ \\
\hline
\end{tabular}

*1 Injected charge $20 \mathrm{nC}$, asymmetrical 1:4 ratio, passive discharge time $0.45 \mathrm{~ms}$

${ }^{* 2}$ Charge error $1.056 \mathrm{nC}$, symmetrical pulses, passive discharge time $5.56 \mathrm{~ms}$.

*3 Injected charge $18.75 \mathrm{nC}$, symmetrical pulses at $0.5 / 1 \mathrm{kHz}$, passive discharge between pulses (calibrated I-DAC).

${ }^{* 4}$ Injected charge $200 \mathrm{nC}$, symmetrical pulses at $1 \mathrm{kHz}$, passive discharge between pulses.

${ }^{* 5}$ Injected charge $100 \mathrm{nC}$, symmetrical pulses at $1 \mathrm{kHz}$, anodic modulation and passive discharge between pulses.

** Static power consumption when the stimulator is in idle state.

electrode voltage and stimulus current characteristics are shown in Fig. 13(b). With passive discharge the measured residual $\Delta Q_{r}$ was $39 \mathrm{pC}$, equivalent to a residual $i_{\mathrm{dc}}$ of $3.9 \mathrm{nA}$ (at $100 \mathrm{~Hz}$ pulse frequency), i.e., a $96 \%$ charge reduction. It is well below the $<100$ nA safety limit [29].

\section{Power Management}

The power supplies and reference voltages were generated as shown in Fig. 1. HVCC was $20 \mathrm{~V}$ using a boost dc-dc converter IC (LM27313) from a $3.7 \mathrm{~V}$ battery. LVCC $(1.8 \mathrm{~V})$ and the $3.3 \mathrm{~V}$ supply for the amplifier $A_{1}$ in Fig. 4 used standard LDOs. The $10 \mathrm{~V}$ reference voltage $\left(V_{\text {ref }}\right)$ was derived from HVCC using MAX6043. The measured voltage output compliance of the stimulator was $18.2 \mathrm{~V}$.

The total power consumption of the LM27313, LDOs and MAX6043 is $43 \mathrm{~mW}$. The total power consumption of the CTDAC (using $250 \mathrm{nA}$ charging current per branch during the charging periods), and opmaps $A_{0}$ and $A_{1}$ in the stimulator chip is $23.3 \mu \mathrm{W}$. The power consumption of the rest of the stimulator circuits (see Fig. 4) is dynamically set depending on the required stimulus current.

For the digital functions, the stimulator is controlled by an FPGA (Digilent Cmod S7). The FPGA receives commands from a PC via UART communication. It also provides the SPI commands, the $1 \mathrm{MHz}$ clock for the chip operation and the 15 $\mathrm{MHz}$ clock for CT-DAC charging.

\section{Comparison and Discussion}

Table I compares the stimulator in this work with other integrated neural stimulators. The CT-DAC principle provides a new alternative to the conventional I-DAC. It offers high resolution with simple layout requirements, and good power efficiency. Unlike most of the stimulators in Table I which have $<8$-bit I-DACs, the stimulator in this work achieves about 10bit resolution while operating in a power efficient manner.

In terms of silicon area, the design in [15], [16] use HV transistors to form the I-DAC at the output stage. Although requiring lower static power consumption, a large silicon area is required. The LV design in [22] with a $3.3 \mathrm{~V}$ output stage occupies $0.064 \mathrm{~mm}^{2}$ but can only be used with low impedance electrodes. Although not in Table I, for a direct comparison, the stimulator in [30] with an 11-bit I-DAC implemented using the same technology consumes $576 \mu \mathrm{A}$ (at maximum I-DAC output) whereas the CT-DAC design (including the voltage-tocurrent conversion and intermediate mirroring stage) consumes about $69 \mu \mathrm{A}$. The I-DAC in [30] occupies $0.11 \mathrm{~mm}^{2}$ compared to $0.027 \mathrm{~mm}^{2}$ used by the CT-DAC with much less layout effort.

The CT-DAC design defines a $Q_{\mathrm{LSB}}$ for charging that is 4.76 times the worst-case switch charge injection $\Delta Q_{d S_{1}}$ as described in Section III-B. This margin required $25 \mathrm{pF}$ capacitors $\left[C_{1}, C_{2}\right.$ in Fig. 6(a)]. Minimizing $\Delta Q_{d s_{1}}$ in future designs could reduce the capacitor size significantly. In addition, these MIM capacitors use only the two top metal layers in the six metal CMOS technology employed. In future layout optimization, the capacitors could be placed on top of the other circuits to reduce silicon area [4].

The maximum stimulus current value has been chosen for the targeted application. In the proposed design scaling up the output current is easily accomplished either by reducing the feedback resistor $R_{f}$ or increasing the scaling factor.

Passive discharge was shown to be effective in the intended application. Asymmetrical pulses, which lead to worse charge balancing compared to symmetrical pulses, were tested. Results showed a residual current of $3.9 \mathrm{nA}$ which is comparable to other work and is well below the safety limit $(<100 \mathrm{nA})$. Potential calibration measures can be employed to improve charge balancing at the expense of reduced CT-DAC bit resolution. 


\section{CONCLUSION}

A 16-channel vagus nerve stimulator using a novel time-tocurrent based design has been presented. Using a small constant dc current of $250 \mathrm{nA}$, the proposed CT-DAC can provide biphasic stimulus currents of up to $550 \mu \mathrm{A}$ with about 10-bit resolution. The stimulator is power efficient, dissipating a static power of $23.3 \mu \mathrm{W}$. It has an output voltage compliance of $18.2 \mathrm{~V}$. A dc residual current of $3.9 \mathrm{nA}$ was recorded with a microelectrode array in saline solution. The novel design is simple, accurate, and its architecture is scalable for multichannel stimulator applications.

\section{ACKNOWLEDGEMENTS}

The authors would like to thank Dr. Ivo Strauss at the Scuola Superiore Sant'Anna, Italy, for providing the microelectrode array.

\section{REFERENCES}

[1] B. Olshansky, H. N. Sabbah, P. J. Hauptman, and W. S. Colucci, "Parasympathetic nervous system and heart failure pathophysiology and potential implications for therapy," Circulation, vol. 118, pp. 863-871, 2008.

[2] C. E. Larson and E. Meng, "A review for the peripheral nerve interface designer," J. Neurosci. Methods, vol. 332:108523, 2020.

[3] I. Delgado-Martínez et al., "Fascicular nerve stimulation and recording using a novel double-aisle regenerative electrode," $J$. Neural Eng., vol. 14, no. 4, 2017.

[4] Y. Wu, D. Jiang, N. Neshatvar, and A. Demosthenous, "A power efficient time-to-current stimulator for vagal-cardiac connection after Heart transplantation," in Proc. IEEE Int. Symp. Circuits Syst. (ISCAS), 2021, pp. 1-4.

[5] E. J. Tehovnik, "Electrical stimulation of neural tissue to evoke behavioral responses," J. Neurosci. Methods, vol. 65, no. 1, pp. 1-17, 1996.

[6] R. Shulyzki et al., "320-channel active probe for high-resolution neuromonitoring and responsive neurostimulation," IEEE Trans. Biomed. Circuits Syst., vol. 9, no. 1, pp. 34-49, 2015.

[7] X. Liu, A. Demosthenous, and N. Donaldson, "An integrated implantable stimulator that is fail-safe without off-chip blockingcapacitors," IEEE Trans. Biomed. Circuits Syst., vol. 2, no. 3, pp. 231-244, 2008.

[8] M. Azin, D. J. Guggenmos, S. Barbay, R. J. Nudo, and P. Mohseni, "A battery-powered activity-dependent intracortical microstimulation IC for brain-machine-brain interface," IEEE $J$. Solid-State Circuits, vol. 46, no. 4, pp. 731-745, 2011.

[9] E. Pepin, D. Micheletti, S. Perlmutter, and J. C. Rudell, "Highvoltage compliant, capacitive-load invariant neural stimulation electronics compatible with standard bulk-CMOS integration," in Proc. IEEE Biomed. Circuits Syst. Conf. (BioCAS), 2014, pp. 260263.

[10] Y. Liu et al., "Bidirectional bioelectronic interfaces: System design and circuit implications," IEEE Solid-State Circuits Mag., vol. 12, no. 2, pp. 30-46, 2020.

[11] M. K. Kim, H. Jeon, H. J. Lee, and M. Je, "Plugging electronics into minds: Recent trends and advances in neural interface microsystems," IEEE Solid-State Circuits Mag., vol. 11, no. 4, pp. 29-42, 2019.

[12] J. P. Uehlin et al., "A single-chip bidirectional neural interface with high-voltage stimulation and adaptive artifact cancellation in standard CMOS," IEEE J. Solid-State Circuits, vol. 55, no. 7, pp. $1749-1761,2020$.

[13] C.-C. Hsieh and M.-D. Ker, "Monopolar biphasic stimulator with discharge function and negative level shifter for neuromodulation SoC integration in low-voltage CMOS process," IEEE Trans. Biomed. Circuits Syst., vol. 15, no. 3, pp. 568-579, 2021.

[14] I. Williams and T. G. Constandinou, "An energy-efficient, dynamic voltage scaling neural stimulator for a proprioceptive prosthesis," IEEE Trans. Biomed. Circuits Syst., vol. 7, no. 2, pp. 129-139, 2013.

[15] A. Taschwer, N. Butz, M. Köhler, D. Rossbach, and Y. Manoli, "A charge balanced neural stimulator with $3.3 \mathrm{~V}$ to $49 \mathrm{~V}$ supply compliance and arbitrary programmable current pulse shapes," in Proc. IEEE Biomed. Circuits Syst. Conf. (BioCAS), 2018, pp. 1-4.

[16] N. Butz, A. Taschwer, S. Nessler, Y. Manoli, and M. Kuhl, "A 22 V compliant $56 \mu \mathrm{W}$ twin-track active charge balancing enabling 100\% charge compensation even in monophasic and $36 \%$ amplitude correction in biphasic neural stimulators," IEEE J. Solid-State Circuits, vol. 53, no. 8, pp. 2298-2310, 2018.

[17] D. Jiang, D. Cirmirakis, and A. Demosthenous, "A vestibular prosthesis with highly-isolated parallel multichannel stimulation," IEEE Trans. Biomed. Circuits Syst., vol. 9, no. 1, pp. 124-137, 2015.

[18] K. Chen, Y.-K. Lo, and W. Liu, "A 37.6mm $\mathrm{mm}^{2}$ 1024-channel highcompliance-voltage SoC for epiretinal prostheses," in Dig. Tech. Papers IEEE Int. Solid-State Circuits Conf. (ISSCC), 2013, pp. 294295.

[19] E. Noorsal, K. Sooksood, H. Xu, R. Hornig, J. Becker, and M. Ortmanns, "A neural stimulator frontend with high-voltage compliance and programmable pulse shape for epiretinal implants," IEEE J. Solid-State Circuits, vol. 41, no. 1, pp. 244-256, 2012.

[20] J. S. Park et al., "1024-pixel CMOS multimodality joint cellular sensor/stimulator array for real-time holistic cellular characterization and cell-based drug screening," IEEE Trans. Biomed. Circuits Syst., vol. 12 , no. 1, pp. 80-94, 2018.

[21] N. Tran et al., "A complete 256-electrode retinal prosthesis chip," IEEE J. Solid-State Circuits, vol. 49, no. 3, pp. 751-765, 2014.

[22] E. Greenwald et al., "A CMOS current steering neurostimulation array withintegrated DAC calibration and charge balancing," IEEE Trans. Biomed. Circuits Syst., vol. 11, no. 2, pp. 324-335, 2017.

[23] D. R. Merrill, M. Bikson, and J. G. R. Jefferys, "Electrical stimulation of excitable tissue: Design of efficacious and safe protocols," $J$. Neurosci. Methods, vol. 141, no. 2, pp. 171-198, 2005.

[24] D. Jiang, A. Demosthenous, T. Perkins, X. Liu, and N. Donaldson, "A stimulator ASIC featuring versatile management for vestibular prostheses," IEEE Trans. Biomed. Circuits Syst., vol. 5, no. 2, pp. $147-159,2011$

[25] D. Jiang and A. Demosthenous, "A multichannel high-frequency power-isolated neural stimulator with crosstalk reduction," IEEE Trans. Biomed. Circuits Syst., vol. 12, no. 4, pp. 940-953, 2018.

[26] J.-Y. Son and H.-K. Cha, "An implantable neural stimulator IC with anodic current pulse modulation based active charge balancing," IEEE Access, vol. 8, pp. 136449-136458, 2020.

[27] M. Ghovanloo and K. Najafi, "A compact large voltage-compliance high output-impedance programmable current source for implantable microstimulators," IEEE Trans. Biomed. Eng., vol. 52, no. 1, pp. 97105, 2005.

[28] G. A. McCallum et al., "Chronic interfacing with the autonomic nervous system using carbon nanotube (CNT) yarn electrodes," Sci. Rep., vol. 7, no. 1, p. 11723, 2017.

[29] J.-J. Sit and R. Sarpeshkar, "A low-power blocking-capacitor-free charge-balanced electrode-stimulator chip with less than $6 \mathrm{nA}$ DC error for 1-mA full-scale stimulation," IEEE Trans. Biomed. Circuits Syst., vol. 1, no. 3, pp. 172-183, 2007.

[30] D. Jiang et al., "An integrated bidirectional multi-channel optoelectro arbitrary waveform stimulator for treating motor neurone disease," in Proc. IEEE Int. Symp. Circuits Systems (ISCAS), 2021, pp. $1-4$. 